\title{
Criatividade e Dificuldade de Aprendizagem: Avaliação com Procedimentos Tradicional e Assistido ${ }^{1}$
}

\author{
Tatiane Lebre Dias ${ }^{2}$ \\ Universidade do Estado de Mato Grosso \\ Sônia Regina Fiorim Enumo \\ Universidade Federal do Espírito Santo
}

\begin{abstract}
RESUMO - Avaliaram-se os efeitos de um programa de criatividade em alunos da $2^{\mathrm{a}}$ e $3^{\mathrm{a}}$ séries de escola pública, com dificuldade de aprendizagem, por combinação de procedimentos tradicional e assistido, nas áreas acadêmica (Teste de Desempenho Escolar- TDE), cognitiva (WISC, Raven e Jogo de Perguntas de Busca com Figuras Diversas- PBFD) e da criatividade (Torrance Verbal e Figurativo). Pelo desempenho no TDE (inferior) e WISC (QI: 92), foi composta a amostra de 34 alunos (8-12 anos), dividida aleatoriamente em dois grupos, sendo G1 submetido a programa de criatividade por 3 meses. No pré-teste, não havia diferenças intergrupos significativas, exceto no Raven, com melhor desempenho de G2; diferença que desapareceu no pós-teste. Neste, houve diferença significativa para G1 no TDE. No PBFD, G1 manteve o desempenho após ajuda, com transferência de aprendizagem e aumentou o perfil alto-escore e transferidor; também melhorou em criatividade verbal (fluência e flexibilidade), enquanto G2 melhorou em flexibilidade.
\end{abstract}

Palavras-chave: criatividade; dificuldade de aprendizagem; avaliação psicológica.

\section{Creativity and Learning Difficulty: Assessment with Traditional and Assisted Procedures}

\begin{abstract}
The effects of a creativity program in 2nd and 3rd grade students from the public school with learning difficulties were evaluated by the combination of assisted and traditional procedures, in the academic area (School Performance Test - SPT), in the cognitive area (WISC, Raven and Constraint - Seeking Questions of Several Questions - C-SQ) and in creativity (Verbal and Figurative Torrance). Considering the performance in the SPT (below average) and WISC (Q.I.: 92), a sample of 34 students (8-12 years old) was formed, divided, at random, into two groups, being the G1 submitted to a creativity program for 3 months. In the pre-test, there were no significant differences among the inter-groups, except in Raven, in which G2 had a better performance; this difference disappeared in the post-test. In this one, there was a significant difference for G1 in the SPT. In the C-SQ, G1 kept the performance after help, with learning transference, and increased the high score and transferable profile; it also improved verbal creativity (fluency and flexibility) whereas G2 improved in flexibility.
\end{abstract}

Key words: creativity; learning difficulty; psychological assessment.

Os problemas enfrentados pelo sistema educacional brasileiro (repetência e evasão escolar, violência nas escolas, por exemplo) tanto podem gerar problemas na aprendizagem dos alunos como agravar as condições das crianças que já apresentam algum tipo de dificuldade de aprendizagem. Nesta mesma perspectiva, o fracasso em tarefas escolares pode conduzir a baixo senso de auto-eficácia para aprendizagem, podendo este ser preditivo para a falta de persistência na aprendizagem de novas tarefas (Torgensen, 1989a).

Em particular, no campo da dificuldade de aprendizagem (DA), há uma heterogeneidade de conceitos que incluem di-

1 Apoio financeiro: Facitec/PMV e CNPq. Esta pesquisa faz parte da tese de doutorado da primeira autora sob orientação da segunda autora. Agradecimentos às bolsistas Flávia Almeida Turini, Maria Júlia de Sá Barbosa e Pereira, Juliana Soares Rabbi, Cláudia Patrocínio Pedroza e Érika da Silva Ferrão, que auxiliaram na coleta e processamento dos dados.

2 Endereço: Avenida 8 de Abril, Residencial 8 de Abril, 331, Bloco 12 Apto 404, Cidade Alta, Cuiabá, MT, Brasil 78025-340. E-mail: t.lebre@uol.com.br ferentes variáveis que podem afetar o desempenho acadêmico (Fonseca, 1995; García, 1998). Face às inúmeras definições, esses autores constataram que tem sido consensualmente adotada a definição proposta pelo National Joint Committee on Learning Disabilities (NJCLD). Nesta definição, destaca-se a importância atribuída às habilidades acadêmicas como explicita Fonseca (1995, p. 71): "Dificuldades de aprendizagem (DA) é um termo geral que se refere a um grupo heterogêneo de transtornos que se manifestam por dificuldades significativas na aquisição e uso da escuta, fala, leitura, escrita, raciocínio ou habilidades matemáticas".

As tentativas de avanços no campo conceitual da DA expandem-se também para o processo de avaliação. Este processo ainda apresenta problemas: existe na área um reconhecimento da falta de uma avaliação confiável da DA (Fonseca, 1995), e uma significativa divergência quanto ao uso do QI para avaliar a DA (Almeida, 1996; Graham \& Harris, 1989; Siegel, 1989; Torgensen, 1989b).

Além desses aspectos conceituais e avaliativos da DA, há também aqueles relacionados ao desempenho apresentado pela criança e na percepção desse desempenho por parte dos 
pais, professores e da própria criança, o qual, geralmente, é associado a baixas expectativas de sucesso (Medeiros, Loureiro, Linhares \& Marturano, 2000; Neves \& Almeida, 1996). Assim, para essas crianças, é reservada pouca margem de sucesso na aprendizagem ou mesmo de talento criativo a ser desenvolvido.

Se na área da DA observam-se grandes dificuldades teórico-metodológicas, também a área da criatividade é histórica e conceitualmente marcada por uma diversidade de enfoques, tanto que Alencar (1995) considera que não existe ainda acordo sobre o significado exato do termo. Contudo, na base das definições, compartilha-se a idéia de que criatividade refere-se à "produção de algo novo e valioso para uma solução" a partir do contexto sócio-histórico-cultural, econômico e lingüístico da sociedade, como defende Lubart (1994), que considera duas características como centrais: a novidade e o propósito do produto criado. Torrance (1965/1976) enfatiza a existência de alguns aspectos cognitivos, considerando o pensamento criativo “(...) como o processo de perceber lacunas ou elementos faltantes, perturbadores; formar idéias ou hipóteses a respeito deles; testar essas hipóteses; e comunicar os resultados, possivelmente modificando e retestando as hipóteses" (p. 34). Cabe também ressaltar as investigações que ressaltam a participação do ambiente como fator de desenvolvimento da criatividade (Alencar, 1995; Torrance, 1965/1976). Nessa perspectiva, especial atenção é dispensada para a influência exercida pela família e pela escola na expressão do comportamento criativo (Alencar, 1995; Bonamigo, 1980; Guilford, 1968, Torrance, 1965/1976).

Dentre os instrumentos de avaliação da criatividade, destacam-se: testes de pensamento divergente, inventários de atitudes e interesses, de personalidade e biográficos, nomeação por professores, pares e supervisores, julgamento de produto, auto-registro de atividades e realizações criativas (Alencar, 1996; Eysenck, 1999; Lubart, 1994). Entretanto, uma forma comumente utilizada para conhecer as habilidades criativas, e ao mesmo tempo, proporcionar um desenvolvimento dessas habilidades pode ser observada nos programas de treinamento da criatividade. Esses, mediante diferentes usos de técnicas e materiais instrucionais, visam a facilitar a expressão da criatividade. No país, investigações têm apontado a importância dos programas de criatividade em diferentes populações (Alencar, 1975; Pereira, 1996; Wechsler, 1987). Os efeitos do programa de criatividade também foram observados por Pollack, Pollack e Tuffli (1973) e Jaben (1986) ao constatarem a presença de habilidades criativas em crianças com déficits cognitivos.

O desenvolvimento da área de avaliação da DA e da criatividade nos conduz a investigar outras possibilidades de avaliação que possam melhor demonstrar o funcionamento do aprendiz. Nesse contexto, tem sido desenvolvida nos últimos 30 anos uma nova abordagem de avaliação denominada "avaliação assistida", "dinâmica" ou "interativa", que visa a detectar potencialidades cognitivas, especialmente em indivíduos portadores de necessidades educativas especiais (NEE) (Sternberg \& Grigorenko, 2002).

O paradigma da avaliação assistida baseia-se em uma das interpretações possíveis do conceito de "zona de desenvolvimento proximal" e de "aprendizagem mediada", propostos por Vygotsky (1991), e no conceito de "experiência de aprendizagem mediada" (mediated learning experienceMLE) de Reuven Feuerstein (Linhares, 1995; Sternberg \& Grigorenko, 2002). Assim, propõe-se a análise da diferença do desempenho da criança sem e com ajuda, no formato testetreino-reteste para pesquisa, também avaliando a quantidade e o tipo de ajuda oferecido pelo mediador.

A avaliação assistida, como explica Tzuriel (2001, p. 6), “(...) refere-se a uma avaliação do pensamento, da percepção, da aprendizagem e da solução de problema por um processo de ensino ativo dirigido para modificar o funcionamento cognitivo". Desse modo, o objetivo da avaliação assistida é identificar o desempenho potencial. Assim, durante o processo de avaliação, inclui-se a assistência do examinador ou mediador, feita por meio do fornecimento de pistas, instrução passo-a-passo, demonstração, sugestão etc (Linhares, 1995).

A importância da mediação foi observada por Burns, Delclos, Vye e Sloan (1996) ao constatarem que as estratégias cognitivas de crianças com deficiências e crianças normais em situação de avaliação mediacional foram significativamente melhores quando comparadas à avaliação tradicional. Efeitos da mediação também foram constatados por Avraham e Ângelo (1996), Burns, Vye, Bransford, Delclos e Ogan (1987) e Carlson e Wiedl (1978). A avaliação assistida também tem sido utilizada na avaliação de programas de educação cognitiva, no qual tem-se verificado maiores escores do grupo experimental que do grupo controle, entre o pré e o pós-teste (Tzuriel, 2001). No Brasil, usando a abordagem de avaliação cognitiva assistida, destacam-se os trabalhos de M.B.M. Linhares e colaboradores enfocando principalmente crianças com dificuldades de aprendizagem (Santa Maria \& Linhares, 1999; Ferriolli, Linhares, Loureiro \& Marturano, 2001).

Com base no exposto acima, considerando: a) ser possível que a criança com DA apresente certas habilidades criativas, b) que a expressão dessas habilidades de alguma maneira se relaciona com habilidades cognitivas e c) que a maneira mais adequada de identificar, estimular e analisar esses aspectos seria por meio de programas de criatividade, questiona-se: o uso de instrumentos num enfoque assistido é adequado para a avaliação de resultados da aplicação de programas de intervenção em habilidades criativas/cognitivas em crianças com DA?

A fim de responder o questionamento acima, esta pesquisa avaliou os efeitos de um programa de promoção da criatividade em crianças com DA, freqüentando as séries iniciais de uma escola pública de uma capital da região sudeste, por meio de uma combinação de procedimentos tradicional e assistido, nas áreas acadêmica, cognitiva e da criatividade.

\section{Metodologia}

\section{Participantes}

A amostra foi composta por 34 alunos (idade média: 9a $4 \mathrm{~m})$ com DA, freqüentando a $2^{\mathrm{a}}$ série (idade média: $9 \mathrm{a} 2 \mathrm{~m}$ ) e $3^{\mathrm{a}}$ série (idade média: 9a 7m) do Ensino Fundamental de uma escola pública de Vitória-ES. A seleção da amostra foi realizada a partir de tratamento estatístico que avaliou a variável "escore bruto total", obtida no Teste de Desem- 
penho Escolar (Stein, 1994) aplicado a 144 alunos da $2^{\mathrm{a}} \mathrm{e}$ $3^{\text {a }}$ séries. Foram selecionados 42 alunos com desempenho dentro de duas faixas (inferior e médio-inferior), sendo 22 alunos da $2^{\mathrm{a}}$ série (pontuação: 78 a 89), a qual corresponde às faixas inferior $(\leq 86)$ e média $(87-105)$ na classificação original do TDE; e 20 alunos da $3^{\text {a }}$ série (pontuação: 92 a $105)$ correspondente no TDE à classificação inferior $(\leq 101)$ e média (102-112).

Posteriormente, realizou-se a avaliação cognitiva através do WISC, sendo que do total de 42 alunos apenas 37 foram avaliados, uma vez que entre essas duas avaliações houve perda de cinco alunos. Dos 37 alunos avaliados pelo WISC, dois foram excluídos por obterem classificação "médio-superior", e um por ter classificação "débilmental". Os demais alunos obtiveram classificação "limítrofe", "médio-inferior" e "média" no WISC, compondo assim, a amostra final de 34 alunos.

Esses alunos foram divididos aleatoriamente em grupo $1-\mathrm{G} 1(\mathrm{n}=17$, idade média $=9 \mathrm{a} 5 \mathrm{~m})\left(2^{\mathrm{a}}\right.$ série $=10$ alunos; $3^{\mathrm{a}}$ série $=7$ alunos $)$; e grupo $2-\mathrm{G} 2(\mathrm{n}=17$, idade média $=9 \mathrm{a} 2 \mathrm{~m})$ ( $2^{\mathrm{a}}$ série $=9$ alunos; $3^{\mathrm{a}}$ série $=8$ alunos). G1 foi submetido a um programa de promoção da criatividade, aplicado em sala de aula da escola, onde também foram feitas as avaliações psicológicas.

\section{Instrumentos}

Com a finalidade de responder aos questionamentos iniciais, foram utilizados na coleta de dados cinco provas psicológicas (quatro provas tradicionais, com caráter psicométrico e aplicação estática, sem ajuda do aplicador, e uma prova assistida, em que o sujeito funciona como seu próprio controle, com medidas antes e após a mediação do aplicador) e um programa de promoção da criatividade.

1) Instrumentos para avaliação e promoção da criatividade:

A) Testes Torrance de Pensamento Criativo - Torrance Tests of Creative Thinking (Torrance, 1990) ${ }^{3}$, visam a avaliar várias habilidades criativas, entre elas, foram aqui avaliadas: a) a fluência, a flexibilidade e a originalidade verbais, e b) a fluência e a originalidade figurativas; sendo aqui aplicados os itens: na Forma Verbal - Aperfeiçoamento do Produto (elefante) e Usos Incomuns (caixas) no pré-teste, e Aperfeiçoamento do Produto (macaco) e Usos Incomuns (latas) no pós-teste. Na Forma Figurativa - Complementação de Figuras (pré e pós-teste), Linhas (pré-teste) e Círculos (pós-teste). B) Programa de promoção da criatividade - teve por objetivo: a) promover o desenvolvimento de habilidades do pensamento criativo (exemplo: uso da imaginação); b) promover o desenvolvimento de habilidades cognitivas no âmbito geral (exemplo: analogias e semelhanças, soluções de problemas); c) desenvolver atitudes afetivo-motivacionais, e d) assegurar a promoção de um ambiente diferenciado do contexto escolar. O programa foi composto por 25 atividades, selecionadas a partir de sugestões contidas

3 Uma adaptação dos testes de Torrance para a população brasileira foi realizada por Wechsler (2002), contendo normas para estudantes do Ensino Médio e Superior, não indicada para esta pesquisa, dada a faixa etária estudada. nos trabalhos de Alencar (2000), Virgolim, Fleith e Pereira (1999) e Wechsler (1998), sendo desenvolvidas em grupo ou individualmente.

2) Instrumentos tradicionais: A) Teste de Desempenho Escolar - TDE (Stein, 1994), instrumento psicométrico fundamentado em critérios elaborados a partir da realidade brasileira que avalia as capacidades fundamentais para o desempenho escolar (Escrita, Aritmética, Leitura e a categoria Total); B) Escala de Inteligência Wechsler

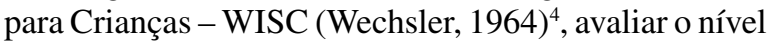
intelectual dos indivíduos, sendo dividido em dois subgrupos - Verbal e Execução; C) Matrizes Progressivas Coloridas de Raven - Escala Especial - MPC (Angelini, Alves, Custódio, Duarte \& Duarte, 1999), teste de inteligência não-verbal padronizado para a população brasileira. As MPC avaliam mais especificamente o fator "g" geral da inteligência, por meio de itens de relações analógicas tradicionais.

3) Prova assistida: A) Jogo de Perguntas de Busca com Figuras Diversas - PBFD -, elaborado por Gera e Linhares (1998). É constituído por 168 desenhos de figuras coloridas, organizadas em 21 arranjos de oito figuras (cada), sendo os arranjos: a) figuras geométricas, b) flores, c) homens, d) cachorros, e) sorvetes, f) meios de transportes, g) casas e h) talheres. O jogo visa a investigar as estratégias utilizadas pelas crianças na elaboração de questões de busca de informação com restrição de alternativas em situação de resolução de problemas. A criança deverá adivinhar qual a figura de cada arranjo o examinador selecionou como figura-alvo, por meio de formulação de perguntas de busca e por raciocínio de exclusão de alternativa, podendo o examinador responder apenas sim ou não. O jogo está dividido em quatro fases: fase inicial sem ajuda (SAJ - quatro arranjos) - sem ajuda do examinador, de modo a avaliar o desempenho real; fase de assistência (ASS - oito arranjos) - recebe-se ajuda do examinador, visando a avaliar o desempenho potencial; fase de manutenção (MAN - quatro arranjos) - suspende-se a ajuda a fim de se saber se as estratégias de perguntas e o raciocínio de exclusão se mantêm; fase de transferência (TRF - quatro arranjos) - avalia a generalização da aprendizagem quanto ao uso de estratégias de perguntas de busca com raciocínio de exclusão. No jogo, as perguntas podem ser do tipo: relevante (especifica um dos atributos dos estímulos, ex: $O$ cachorro está com orelha para cima?), irrelevante (menciona atributos desnecessários, ex: É grande? Após ter perguntado se era pequeno), repetida (repete pergunta já formulada), incorreta (menciona um atributo inespecífico, ex: Qual é a cor?).

\section{Procedimento}

A pesquisa foi realizada nas seguintes etapas: 1 ) pré-teste: após seleção da amostra feita pelos resultados do TDE e do WISC (novembro-dezembro, 2001), aplicou-se as Matrizes Progressivas Coloridas de Raven - Escala Especial (fevereiro, 2002), em grupos de oito alunos, pela pesquisadora. Posteriormente, realizou-se a avaliação da criatividade atra-

4 Não estava disponível à época da pesquisa a versão brasileira atualizada do WISC-III. 
vés dos Testes Torrance de Pensamento Criativo (fevereiro, 2002), aplicado em duas sessões em grupos de 10 alunos. E, por último, aplicou-se o PBFD, individualmente, sendo as sessões filmadas por uma bolsista de IC/CNPq; 2) intervenção: aplicou-se o programa de promoção da criatividade em 25 sessões, executadas três vezes por semana (abril-junho, 2002), com tempo médio de 60 minutos, sendo uma sessão no turno matutino ( $8-9 \mathrm{~h}$ da manhã) com nove alunos (quatro meninos), no vespertino (14-15 h) com oito alunos (quatro meninos). As sessões foram filmadas por uma bolsista de IC/ CNPq; 3) pós-teste: os alunos foram reavaliados seguindo a mesma ordem e procedimento de aplicação dos instrumentos (TDE, WISC, Raven, Testes Torrance de Pensamento Criativo e PBFD), nos meses (junho-julho, 2002), com auxílio de bolsistas de IC/CNPq.

\section{Processamento e análise dos dados}

O tratamento estatístico foi processado pelo software SPSS 7.0, com auxílio de estatísticos. Os instrumentos foram assim processados:

1) Instrumentos tradicionais: a) Testes Torrance de Pensamento Criativo: após os testes serem corrigidos por três juízes independentemente, selecionou-se aleatoriamente 20 aplicações ( 10 do pré e 10 do pós-teste, sendo cinco do G1 e cinco do G2 em cada etapa) para o cálculo do Índice de Concordância (IC), não sendo obtido índice inferior a 70\%; b) Teste de Desempenho Acadêmico: a correção e análise seguiram os critérios de classificação do próprio teste; c) WISC: a correção e análise do WISC seguiram os critérios contidos no próprio teste; d) Raven (MPC): a correção e análise também seguiram os critérios do teste.

2) Prova assistida: a partir dos dados obtidos quanto aos tipos de perguntas de busca e quanto aos tipos de tentativas de solução no PBFD, obteve-se o perfil de desempenho cognitivo dos alunos no G1 e G2, no pré e no pós-teste. As tentativas de solução podem ser: correta, incorreta e correta ao acaso. Após o cálculo das proporções de perguntas de busca (relevante, irrelevante, incorreta e repetida) e de tentativas de solução (correta, incorreta e correta ao acaso) em cada fase do jogo (SAJ, ASS, MAN e TRF), obtém-se o Perfil de Desempenho Cognitivo ${ }^{5}$ quanto à eficiência e manutenção da aprendizagem, que pode ser: alto-escore, ganhador, não-mantenedor; transferidor ou não- transferidor.

Para avaliar se foram significativas as diferenças de desempenho dos alunos entre o pré e o pós-teste, aplicou-se o Teste de $t$ pareado (diferenças intragrupo). Para verificar

5 Critérios para os perfis de desempenho no PBFD: alto-escore (presença de 0,70 de perguntas relevantes e 0,75 de soluções corretas, na fase SAJ; e pelo menos 0,60 de perguntas relevantes e 0,60 de soluções corretas, na fase MAN); ganhador (presença de pelo menos 0,60 de perguntas relevantes e 0,50 de soluções corretas, na fase MAN); não-mantenedor (presença de menos de 0,60 de perguntas relevantes na fase SAJ e/ou menos de 0,50 de soluções corretas, na fase MAN), transferidor (presença de 0,60 de perguntas relevantes e 0,60 de solução correta, na fase TRF), não transferidor (presença de menos de 0,60 de perguntas relevantes e menos de 0,60 de solução correta, na fase de TRF). se houve diferença significativa de desempenho intergrupos (G1 versus G2), utilizou-se o teste $t$ de Student. Na prova assistida (PBFD), usou-se o teste de Wilcoxon para avaliar a significâncias das diferenças de desempenho entre as fases.

Para garantir a participação na pesquisa, foi assinado pelo pai ou responsável de cada participante um termo de autorização contendo explicações dos objetivos e condições de participação no projeto, após aprovação da pesquisa pelo Comitê de Ética do Programa de Pós-Graduação em Psicologia da universidade. A pesquisa não trouxe riscos físicos e psicológicos para os alunos, tendo utilizado material atóxico, sendo os resultados apresentados aos professores.

\section{Resultados}

O desempenho médio dos grupos nas provas tradicionais, não assistidas, nas áreas acadêmica, cognitiva e da criatividade, no pré e pós-teste, pode ser visualizado na Tabela 1.

A Tabela 2 mostra a significância estatística das diferenças no desempenho de cada grupo entre o pré-teste e o pós-teste, e do desempenho entre os grupos no pré e pós-teste na avaliação tradicional, não-assistida, nas áreas acadêmica, cognitiva e da criatividade.

\section{Avaliação do desempenho acadêmico}

Na avaliação acadêmica pelo TDE, os alunos do G1 apresentaram uma melhora geral do desempenho (Tabela 1), a qual foi estatisticamente significativa nas áreas de Escrita e Aritmética e no desempenho Total, após o período de escolarização e aplicação do Programa de Promoção da Criatividade (Tabela 2). Este desempenho de G1 contrasta com de G2, que melhorou significativamente apenas no subteste de Escrita (Tabela 2). Assim, os grupos não se diferenciaram no pré e no pós-teste do TDE, mas apresentaram diferenças intragrupo entre o pré e o pós-teste (Tabela 2).

\section{Avaliação do desempenho cognitivo - WISC}

Na avaliação cognitiva realizada pelo WISC, vê-se, na Tabela 1, que os grupos apresentaram padrões similares, isto é, eram semelhantes no pré-teste, compondo, intencionalmente, uma amostra homogênea, e que melhoraram o desempenho no QI-Execução e QI-Total no pós-teste, com um aumento médio de 6 pontos no QI-Total e de 7 pontos no QI-Execução. Também houve aumento de 4 pontos no QI-Verbal para os dois grupos; porém, este aumento não foi estatisticamente significativo (Tabela 2). Assim, inicialmente, não havia diferenças significativas entre os dois grupos quanto ao QI-Total (ambos com QI médio de 92,6) (Tabela 1). Eles mantiveram esta semelhança no pós-teste, com QI médio de 98 (Tabela 1). Entretanto, entre o pré e o pós-teste, os grupos apresentaram diferenças significativas no QI-Execução e no QI-Total, destacando-se que as diferenças no QI-Total do G1 foram significativas no nível de 0,01 (Tabela 2).

\section{Avaliação do desempenho cognitivo - Raven (MPC)}

Complementando a avaliação cognitiva, utilizou-se um teste não-verbal, que exige aplicação do raciocínio analógico 
Tabela 1. Média e desvio-padrão dos grupos na avaliação tradicional (acadêmica, cognitiva e da criatividade), no pré-teste e pós-teste.

\begin{tabular}{|c|c|c|c|c|c|c|c|c|}
\hline \multirow{3}{*}{$\begin{array}{c}\text { Grupos } \\
\text { Fase } \\
\text { Provas }\end{array}$} & \multicolumn{4}{|c|}{ G1 } & \multicolumn{4}{|c|}{ G2 } \\
\hline & \multicolumn{2}{|c|}{ Pré } & \multicolumn{2}{|c|}{ Pós } & \multicolumn{2}{|c|}{ Pré } & \multicolumn{2}{|c|}{ Pós } \\
\hline & M & SD & $\mathbf{M}$ & SD & $\mathbf{M}$ & SD & M & SD \\
\hline \multicolumn{9}{|l|}{ Acadêmica-TDE } \\
\hline Escrita & 17.71 & 4.63 & 20.65 & 5.01 & 17.29 & 4.41 & 20.94 & 4.72 \\
\hline Aritmética & 10.12 & 3.10 & 13.35 & 3.67 & 11.29 & 3.44 & 12.82 & 4.08 \\
\hline Leitura & 62.65 & 4.62 & $60 ; 29$ & 5.02 & 62.53 & 2.45 & 61.06 & 4.15 \\
\hline Total & 90.47 & 8.58 & 94.29 & 11.03 & 91.47 & 6.87 & 94.82 & 6.87 \\
\hline \multicolumn{9}{|l|}{ Cognitiva-WISC } \\
\hline QI-Verbal & 97.29 & 8.02 & 101.29 & 11.74 & 96.94 & 8.13 & 101.18 & 8.15 \\
\hline QI-Execução & 88.53 & 11.95 & 95.24 & 10.82 & 88.82 & 11.63 & 95.06 & 14.91 \\
\hline QI-Total & 92.65 & 9.51 & 98.24 & 8.85 & 92.65 & 9.51 & 98.41 & 10.68 \\
\hline Cognitiva-Raven & 47.94 & 22.54 & 60.29 & 23.08 & 66.33 & 22.51 & 68 & 17.20 \\
\hline \multicolumn{9}{|l|}{ Criativ.Verbal } \\
\hline Fluência & 15.41 & 6.63 & 21.41 & 6.18 & 15.59 & 5.5 & 19.06 & 8.47 \\
\hline Flexibilidade & 8.58 & 2.69 & 11.12 & 3.41 & 7.76 & 1.98 & 10.12 & 2.76 \\
\hline Originalidade & 6.41 & 4.86 & 8.12 & 5.64 & 5.23 & 4.05 & 8.76 & 7.82 \\
\hline \multicolumn{9}{|l|}{ Criativ.Figurativa } \\
\hline Fluência & 16.41 & 3.49 & 18.82 & 6 & 17.35 & 4.78 & 15.94 & 4.35 \\
\hline Originalidade & 8.94 & 3.47 & 10.23 & 5.08 & 9.12 & 2.76 & 9.47 & 4.01 \\
\hline
\end{tabular}

Tabela 2. Significância das diferenças do desempenho intragrupo (pré-teste X pós-teste) e intergrupos (G1 X G2) na avaliação tradicional (acadêmica, cognitiva e da criatividade).

\begin{tabular}{|c|c|c|c|c|}
\hline \multirow{3}{*}{ Comparações } & \multirow{2}{*}{\multicolumn{2}{|c|}{$\frac{\text { Diferenças intragrupo }}{\text { (Teste } t \text { pareado) }}$}} & \multirow{2}{*}{\multicolumn{2}{|c|}{$\begin{array}{c}\text { Diferenças intergrupos } \\
\text { (Teste } t \text { de } \text { Student })\end{array}$}} \\
\hline & & & & \\
\hline & G1 & G2 & Pré & Pós \\
\hline Provas & pré $X$ pós & pré X pós & G1 X G2 & G1 X G2 \\
\hline \multicolumn{5}{|l|}{ Acadêmica TDE } \\
\hline Escrita & $.001732 * *$ & $.00007507 * *$ & .792 & .861 \\
\hline Aritmética & $.000369 *$ & .07410 & .303 & .694 \\
\hline Leitura & .08538 & .2338 & 109 & .632 \\
\hline Total & $.03116^{*}$ & .06484 & .710 & .889 \\
\hline \multicolumn{5}{|l|}{ Cognitiva WISC } \\
\hline QI-Verbal & .0893 & .08831 & .8994 & .9731 \\
\hline QI-Execução & $.01240 *$ & $.03588^{*}$ & .9425 & .9687 \\
\hline QI-Total & $.00425 * *$ & $.02252 *$ & .9403 & .9309 \\
\hline Cognitiva Raven & .07014 & .74 & $.028 *$ & .298 \\
\hline \multicolumn{5}{|l|}{ Criatividade Verbal } \\
\hline Fluência & $.00642 * *$ & .87511 & .933 & .362 \\
\hline Flexibilidade & $.00806^{* *}$ & $.00452 * *$ & .318 & .354 \\
\hline Originalidade & .20055 & .11222 & 449 & .784 \\
\hline \multicolumn{5}{|l|}{ Criativ. Figurativa } \\
\hline Fluência & .24322 & .11222 & .514 & .119 \\
\hline Originalidade & .53321 & .90901 & .871 & .630 \\
\hline
\end{tabular}

${ }^{*} p \leq .05 ; * p \leq 0.01$

- Raven (MPC) (Angelini \& cols., 1999). Como se vê na Tabela 1, os grupos apresentavam desempenhos distintos no pré-teste, favorável ao G2, diferença esta significativa (Tabela 2). Após quatro meses de escolarização para os dois grupos e de intervenção para G1, essa diferença deixou de existir no pós-teste, ocorrendo um expressivo aumento na média do G1 entre o pré e o pós-teste (Tabela 1), porém, não significativo (Tabela 2 ). 


\section{Avaliação da criatividade}

Na Forma Verbal dos Testes Torrance, no pré-teste, as médias dos grupos foram próximas nas seguintes categorias: Fluência, Flexibilidade e Originalidade (Tabela 1). Após o período de intervenção e de escolarização, G1 melhorou significativamente as médias em Fluência e Flexibilidade verbais, ou seja, na capacidade de produzir um maior número de resposta para produção de algo novo ou na mudança de um objeto e também na variedade de categorias em que essas respostas podem ser incluídas (Tabela 2). G2 melhorou apenas em Flexibilidade. Embora ambos os grupos tenham aumentado as médias em Originalidade Verbal, as diferenças não foram significativas (Tabela 2). Mesmo com essa melhora intragrupo, os grupos não apresentaram diferenças significativas entre si, tanto no pré-teste como no pós-teste (Tabela 2).

\section{Prova cognitiva assistida}

A análise do desempenho na prova cognitiva assistida (PBFD) é feita a partir das comparações das fases do jogo (sem ajuda- SAJ, assistência- ASS, manutenção- MAN e transferência- TRF), através da proporção de perguntas de busca (relevantes, irrelevantes, incorretas e repetidas) e do tipo de solução (correta, incorreta e correta ao acaso). Com base nessa análise, obtêm-se os perfis de desempenho no PBFD: alto-escore, ganhador, não-mantenedor, e transferidor ou não-transferidor.

No pré-teste do PBFD, comparando o desempenho dos alunos entre as fases do jogo, observa-se que, no G1, houve um aumento significativo de perguntas relevantes e diminuição também significativa de perguntas incorretas entre as fases (SAJ-MAN), ocorrendo o mesmo entre as fases SAJTRF (Tabela 3). Esta mesma dinâmica em relação ao aumento de perguntas relevantes e diminuições de incorretas também ocorreu no G2, porém, houve um aumento significativo de perguntas irrelevantes entre as fases SAJ-TRF, segundo o teste de Wilcoxon (Tabelas 3 e 4).

Quanto ao tipo de tentativa de solução - PTTS (Tabelas 3 e 4), observou-se, no G1, um aumento significativo de tentativas corretas e diminuição de tentativas incorretas e corretas ao acaso entre as fases SAJ-MAN, SAJ-TRF. No G2, houve um aumento significativo de tentativas corretas

Tabela 3. Indicadores de desempenho (mediana e amplitude de variação) dos grupos na prova assistida (PBFD), no pré-teste.

\begin{tabular}{|c|c|c|c|c|c|c|c|c|c|c|c|c|}
\hline \multirow{3}{*}{$\begin{array}{c}\text { Grupos } \\
\text { Fases }\end{array}$} & \multicolumn{6}{|c|}{ G1 } & \multicolumn{6}{|c|}{ G2 } \\
\hline & \multicolumn{2}{|c|}{ SAJ } & \multicolumn{2}{|c|}{ MAN } & \multicolumn{2}{|c|}{ TRF } & \multicolumn{2}{|c|}{ SAJ } & \multicolumn{2}{|c|}{ MAN } & \multicolumn{2}{|l|}{ TRF } \\
\hline & Md & $\mathbf{A V}$ & Md & $\mathbf{A V}$ & Md & $\mathbf{A V}$ & Md & AV & Md & $\mathbf{A V}$ & Md & $\mathbf{A V}$ \\
\hline $\mathrm{N}^{\circ}$ médio perguntas busca por arranjo & 5 & $2-9.5$ & 3.5 & $2.5-4.7$ & 3.5 & $1.5-5$ & 4 & $2-6.7$ & 3.5 & $2.7-7.2$ & 3.75 & $2.5-8$ \\
\hline \multicolumn{13}{|l|}{ PTPB } \\
\hline Relevante & .38 & $0-.78$ & .91 & $.50-1$ & .8 & $.15-1$ & .64 & $0-.88$ & .99 & $.48-1$ & .85 & $.44-1$ \\
\hline Irrelevante & .07 & $0-.22$ & .06 & $0-.2$ & .07 & $0-.15$ & .11 & $0-.3$ & .06 & $0-.35$ & .07 & $0-.35$ \\
\hline Incorreta & .37 & $0-1$ & .07 & $0-.4$ & .13 & $0-.95$ & .22 & $0-.72$ & 0 & $0-.22$ & .07 & $0-.19$ \\
\hline Repetida & 0 & $0-.33$ & 0 & 0 & 0 & $0-.07$ & 0 & $0-.26$ & 0 & $0-.17$ & 1 & $0-.25$ \\
\hline $\mathrm{N}^{\circ}$ médio perguntas de busca por arranjo & 2 & $1-6.25$ & 1 & $1-2.25$ & 1.25 & $1-4.5$ & 1.25 & $1-4.5$ & 1 & $1-2$ & 1 & $1-1.25$ \\
\hline \multicolumn{13}{|l|}{ PTTS } \\
\hline Correta & .13 & $0-.75$ & 1 & $.14-1$ & .8 & $0-1$ & .75 & $0-1$ & 0.75 & $.25-1$ & 1 & $0-1$ \\
\hline Incorreta & .5 & $0-.85$ & 0 & $0-.56$ & .2 & $0-.78$ & .2 & $0-.8$ & 0 & $0-.5$ & 0 & $0-.2$ \\
\hline Correta-ao-acaso & .21 & $0-.44$ & 0 & $0-.5$ & 0 & $0-.6$ & .17 & $0-.8$ & 0.12 & $0-.25$ & 0 & $0-.8$ \\
\hline
\end{tabular}

PTPB: Proporção dos tipos de pergunta de busca; PTTS: Proporção dos tipos de tentativa de solução; SAJ: sem ajuda; MAN: manutenção; TRF: transferência..

Tabela 4. Comparações do desempenho dos grupos entre as fases da prova assistida (PBFD), no pré-teste.

\begin{tabular}{|c|c|c|c|c|c|c|}
\hline \multirow{2}{*}{$\begin{array}{c}\text { Grupos } \\
\text { Fases }\end{array}$} & \multicolumn{3}{|c|}{ G1 } & \multicolumn{3}{|c|}{ G2 } \\
\hline & SAJ X MAN & SAJ X TRF & MAN X TRF & SAJ X MAN & SAJ X TRF & MAN X TRF \\
\hline $\mathrm{N}^{\circ}$ médio perguntas de busca/ arranjo & $.0052 * *$ & $.0066^{* *}$ & 1 & .4244 & .4933 & .4616 \\
\hline \multicolumn{7}{|l|}{ PTPB } \\
\hline Relevante & $.0084^{* *}$ & $.0018 * *$ & .3455 & $.0005^{* *}$ & $.0016^{* *}$ & .7239 \\
\hline Irrelevante & .8999 & .6486 & .8333 & .0543 & $.0185^{*}$ & .2149 \\
\hline Incorreta & $.0031^{* *}$ & $.0044 * *$ & .1680 & $.0061 * *$ & $.0061^{* *}$ & .6552 \\
\hline $\mathrm{N}^{\circ}$ médio perguntas de busca/ arranjo & $.0008^{* *}$ & $.0021^{* * *}$ & $.0323 *$ & .0655 & $.0091 *$ & .1408 \\
\hline \multicolumn{7}{|l|}{ PTTS } \\
\hline Correta & $.016^{*}$ & $.007 * *$ & .933 & $.026^{*}$ & .100 & .469 \\
\hline Incorreta & $.017^{*}$ & $.005^{* *}$ & .796 & $.017^{*}$ & .049 & .417 \\
\hline Correta-ao- acaso & $.040 *$ & $.028 *$ & .465 & .561 & .891 & .564 \\
\hline
\end{tabular}

$\left({ }^{*} p \leq 0.05 ; * * p \leq 0.01\right.$; teste de Wilcoxon); PTPB: Proporção dos tipos de pergunta de busca; PTTS::Proporção dos tipos de tentativa de solução.. SAJ: sem ajuda MAN: manutenção; TRF: transferência. 
e uma diminuição de tentativas incorretas somente entre as fases SAJ-MAN.

Em resumo, pode-se dizer que, no pré-teste, após a fase de assistência (ASS), ambos os grupos se beneficiaram da ajuda da examinadora, apresentando um aumento na freqüência de perguntas relevantes e de tentativas corretas nas fases de MAN e TRF, e diminuindo o número de perguntas irrelevantes e de tentativas de solução incorretas e corretasao-acaso. Contudo, essa melhora foi mais expressiva no G1, que se beneficiou mais da ajuda da examinadora, dado o aumento significativo de tentativa de solução correta entre as fases SAJ-MAN e SAJ-TRF. No GC, houve um aumento significativo de perguntas irrelevantes.

No pós-teste (Tabelas 5 e 6), no G1, comparando as fases SAJ-MAN do PBFD, nota-se que houve aumento significativo de perguntas relevantes (PTPB); enquanto, no G2, houve uma diminuição significativa desse tipo de pergunta. Quanto às perguntas incorretas (PTTS), nos dois grupos, constatou-se uma diminuição significativa entre as fases SAJ-MAN e SAJ-TRF.

Em relação às tentativas de solução (PTTS), constata-se, na Tabela 6, que houve um aumento significativo de tentativas corretas para G1 entre as fases SAJ-MAN e SAJ-TRF; no
G2, esse aumento ocorreu somente entre as fases SAJ-MAN. As tentativas incorretas também diminuíram significativamente em ambos os grupos (SAJ-MAN, SAJ-TRF). Ainda no G1, observa-se uma diminuição significativa de tentativas corretas ao acaso entre as fases SAJ-MAN e SAJ-TRF (Tabelas 5 e 6).

No pós-teste (Tabelas 5 e 6), nota-se que G1 manteve a ajuda recebida pela examinadora, realizando a transferência da aprendizagem. Esta transferência ficou evidente pelo aumento de perguntas relevantes e pela diminuição de perguntas incorretas entre as fases SAJ-MAN e SAJ-TRF. Por sua vez, G2 teve dificuldade em manter a ajuda recebida pela examinadora, o que ficou evidenciado pela diminuição significativa de perguntas relevantes entre as fases SAJMAN. O melhor desempenho do G1 quanto à eficiência na manutenção e transferência complexa da aprendizagem foi decorrente do aumento significativo de tentativas corretas entre as fases SAJ-MAN e SAJ-TRF; enquanto no G2, esse aumento ocorreu somente entre as fases SAJ-MAN.

A partir dos dados obtidos quanto aos tipos de perguntas de busca e quanto aos tipos de tentativas de solução no PBFD, obteve-se o perfil de desempenho cognitivo dos alunos no pré e no pós-teste, sendo classificados em: alto-escore,

Tabela 5. Indicadores de desempenho (mediana e amplitude de variação) dos grupos na prova assistida (PBFD), no pós-teste.

\begin{tabular}{|c|c|c|c|c|c|c|c|c|c|c|c|c|}
\hline \multirow{3}{*}{$\begin{array}{c}\text { Grupos } \\
\text { Fases }\end{array}$} & \multicolumn{6}{|c|}{ G1 } & \multicolumn{6}{|c|}{ G2 } \\
\hline & \multicolumn{2}{|c|}{ SAJ } & \multicolumn{2}{|c|}{ MAN } & \multicolumn{2}{|c|}{ TRF } & \multicolumn{2}{|c|}{ SAJ } & \multicolumn{2}{|c|}{ MAN } & \multicolumn{2}{|c|}{ TRF } \\
\hline & Md & AV & Md & AV & Md & AV & Md & AV & Md & AV & Md & AV \\
\hline $\mathrm{N}^{\circ}$ médio perguntas de busca/ arranjo & 4.25 & $3.5-10$ & 3.7 & $2.5-5.2$ & 43. & $2-5.2$ & 4.75 & $3-6.5$ & 3.75 & $3-5.5$ & 3.5 & $3-4.75$ \\
\hline \multicolumn{13}{|l|}{ PTPB } \\
\hline Relevante & .76 & $.1-.9$ & .88 & $.7-1$ & .87 & $.62-1$ & .80 & $.4-1$ & .93 & $.88-1$ & .93 & $.63-1$ \\
\hline Irrelevante & .07 & $0-.31$ & .0 & $0-.25$ & .06 & $0-.19$ & .09 & $0-.5$ & .06 & $0-.18$ & .0 & $0-.16$ \\
\hline Incorreta & .12 & $0-.52$ & 0 & $0-.13$ & .0 & $0-.19$ & .12 & $0-.5$ & 0 & $0-.19$ & .06 & $0-.21$ \\
\hline Repetida & 0 & $0-.22$ & 0 & $0-.1$ & 0 & $0-.06$ & 0 & $0-.9$ & 0 & $0-.14$ & 0 & 0 \\
\hline $\mathrm{N}^{\circ}$ médio perguntas de busca/ arranjo & 1.25 & $1-4$ & 1 & $1-1.75$ & 1 & $1-1.75$ & 1.25 & $1-4$ & 1 & $1-1.75$ & 1 & $1-1.75$ \\
\hline \multicolumn{13}{|l|}{ PTTS } \\
\hline Correta & .6 & $0-1$ & 1 & $.43-1$ & 1 & $.43-1$ & .8 & $.27-1$ & 1 & $.6-1$ & 1 & $.5-1$ \\
\hline Incorreta & .2 & $0-.75$ & 0 & $0-.43$ & 0 & $0-.5$ & .2 & $0-.63$ & 0 & $0-.2$ & 0 & $0-.3$ \\
\hline Correta-ao-acaso & .11 & $0-.4$ & 0 & $0-.25$ & 0 & $0-.2$ & .0 & $0-.2$ & 0 & $0-.2$ & 0 & $0-.2$ \\
\hline
\end{tabular}

PTPB: Proporção dos tipos de pergunta de busca; PTTS: Proporção dos tipos de tentativa de solução; SAJ: sem ajuda; MAN: manutenção; TRF: transferência.

Tabela 6. Comparações do desempenho dos grupos entre as fases da prova assistida (PBFD), no pós-teste.

\begin{tabular}{|c|c|c|c|c|}
\hline \multirow{2}{*}{$\begin{array}{c}\text { Grupos } \\
\text { Fases }\end{array}$} & \multicolumn{2}{|c|}{ G1 } & \multicolumn{2}{|c|}{ G2 } \\
\hline & SAJ X MAN & SAJ X TRF & SAJ X MAN & SAJ X TRF \\
\hline $\mathrm{N}^{\circ}$ médio perguntas de busca/ arranjo & $.0211^{*}$ & $.0134^{*}$ & $.0004 * *$ & $.0009 * *$ \\
\hline \multicolumn{5}{|l|}{ PTPB } \\
\hline Relevante & $.0006^{* *}$ & $.0060^{* *}$ & $.0026^{* *}$ & $.0013^{* *}$ \\
\hline Irrelevante & .1202 & .1235 & .2007 & .8496 \\
\hline Incorreta & $.0004 * *$ & $.0023^{* *}$ & $.0024 * *$ & $.0024 * *$ \\
\hline Repetida & $.0117 *$ & .322 & .5002 & .7256 \\
\hline $\mathrm{N}^{\circ}$ médio perguntas de busca/ arranjo & $.0276^{*}$ & $.0045 * *$ & $.0128^{*}$ & $.0554 *$ \\
\hline \multicolumn{5}{|l|}{ PTTS } \\
\hline Correta & $.016^{*}$ & $.007 * *$ & $.028^{*}$ & .106 \\
\hline Incorreta & $.017 *$ & $.005 * *$ & $.017 *$ & .049 \\
\hline Correta-ao-acaso & $.040 *$ & $.028^{*}$ & .581 & .891 \\
\hline
\end{tabular}

$\left({ }^{*} p \leq 0.05 ; * * p \leq 0.01\right.$; teste de Wilcoxon); PTPB: Proporção dos tipos de pergunta de busca; PTTS: Proporção dos tipos de tentativa de solução; SAJ: sem ajuda; MAN: manutenção; TRF: transferência. 
ganhador, ou não-mantenedor. Com base na fase de transferência do PBFD foi possível obter os perfis: transferidor ou não-transferidor.

No pré-teste do PBFD, observa-se na Figura 1 que G2 apresentou maior número de alunos com perfil alto-escore (seis alunos), indicando um bom desempenho desde a fase sem ajuda, fazendo perguntas de busca relevantes e tentativas de solução corretas até o final, na fase de manutenção. G2 apresentou também maior número de alunos com perfil nãomantenedor (desempenho abaixo dos níveis estabelecidos em relação ao perfil ganhador na fase de manutenção). Por sua vez, G1 apresentou maior número de alunos com perfil ganhador (apresenta bom desempenho na fase sem ajuda e, com a assistência, melhora o desempenho na fase de manutenção) (Figura 1).

No pós-teste do PBFD (Figura 1), aumentou o número de alunos com perfil alto-escore em ambos os grupos; entretanto, nota-se que G1 teve um aumento expressivo (sete) no número de alunos com esse perfil, enquanto que, no G2, este aumento foi de quatro alunos. No G1, houve diminuição no perfil não-mantenedor (1) e, no G2, este perfil deixou de existir. Houve uma maior diminuição do número de alunos com perfil ganhador no G1 (6 alunos) do que no G2, que teve uma diminuição de um aluno.

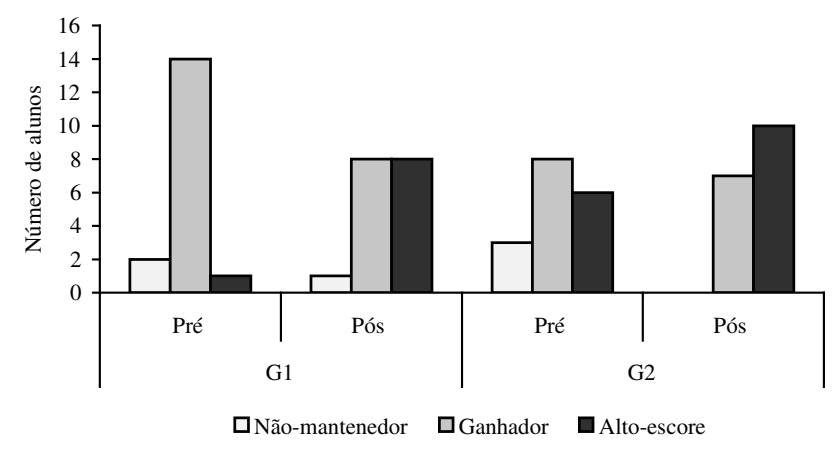

Figura 1. Perfis de desempenho cognitivo dos grupos na prova assistida (PBFD), no pré-teste e pós-teste.

Em resumo, G2 apresentou melhor desempenho no préteste do PBFD; porém, G1 melhorou consideravelmente o seu desempenho no jogo, aproximando do G2 quanto ao número de alunos com perfil alto-escore (Figura 1).

Quanto à transferência de aprendizagem no PBFD, G1 aumentou o número de alunos com perfil transferidor entre pré e o pós-teste (de 10 para 15), aproximando-se do G2, que manteve o mesmo número de alunos entre o pré e o pós-teste com esse perfil (16 alunos).

Em resumo, no que se refere ao processo de transferência de aprendizagem avaliada quanto à eficiência de perguntas de busca com restrição de alternativas no PBFD, nota-se que G2, inicialmente, apresentou melhor desempenho. Contudo, após o programa de promoção da criatividade, G1 se sobressaiu, sendo o grupo que mais se beneficiou da ajuda da examinadora, mantendo o desempenho até a fase de transferência da aprendizagem no jogo; enquanto G2 manteve-se igual.

Finalmente, o aumento de perfil alto-escore e transferidor no G1, ocorrido entre o pré e o pós-teste do PBFD, expressa possíveis efeitos do programa de promoção da criatividade e da escolaridade, uma vez que os grupos apresentavam desempenhos distintos no início do jogo.

\section{Discussão}

Os resultados mostraram o desempenho de dois grupos de alunos em dois procedimentos de avaliação - tradicional e assistido - antes e após intervenção no G1, submetido a um programa de promoção da criatividade por três meses.

Primeiramente, em relação à avaliação acadêmica (TDE), constatou-se o melhor desempenho de G1, podendo-se assim pensar que o período de escolarização, juntamente com o Programa de Promoção da Criatividade, tiveram maior efeito nesse grupo, de modo a alterar o desempenho nas habilidades acadêmicas de alunos com DA. Embora o Programa não tivesse em si um caráter exclusivamente escolar, evidenciou, sobretudo, a suscetibilidade de alunos com dificuldade de aprendizagem (DA) ao programa de intervenção. Diante disso, no que se refere à avaliação de crianças com DA, o uso de uma metodologia de intervenção mostrou-se mais adequado. Tal evidência também é compartilhada por Torgensen (1988), que considera o uso de programas de avaliação/intervenção uma forma de pesquisa aplicada importante no campo da DA, por possibilitar a validação de teorias derivadas de pesquisas básicas, e também por fornecer informações dessas práticas na variedade de locais onde elas são usadas, fornecendo dados contextualizados.

Em relação aos resultados do WISC, o aumento de QI em ambos os grupos e as semelhanças nos dados de G1 e G2, no pré e pós-teste, mostram os efeitos do período de escolarização e as influências das experiências vividas pelas crianças no seu meio social. Nesse sentido, ainda em se tratando da questão da avaliação na área da DA, pode-se verificar que, para alunos com DA, o uso de testes de QI se mostrou adequado, confirmando o posicionamento de Almeida (1996) e Torgensen (1989b), e contrariando o ponto de vista de Grahan e Harris (1989) e Siegel (1989) quanto ao uso de testes de QI para estes casos.

A semelhança inicial entre os grupos nas avaliações acadêmica (TDE) e cognitiva (WISC) deixou de existir na medida em que, no Raven, G2 mostrou um melhor desempenho neste tipo de prova cognitiva logo no pré-teste. No pós-teste, essa diferença deixou de existir, revelando uma melhora acentuada de desempenho do G1. Em outras palavras, os alunos do G1 melhoraram a capacidade para fazer comparações, raciocinar por analogia, atentar melhor para a relação parte-todo. Pode-se dizer que, à medida que se promoveu o comportamento criativo, essas habilidades se fizeram mais presentes em crianças com DA.

A melhora no desempenho acadêmico (TDE) e cognitivo (WISC e Raven) dos alunos, principalmente do G1, chama a atenção para a presença de habilidades criativas em crianças com DA, e ao mesmo tempo, para a capacidade de se desenvolverem tais habilidades. Embora os grupos mantivessem semelhanças entre si nas habilidades avaliadas pelos Testes Torrance de Pensamento Criativo, internamente os grupos melhoraram entre o pré e o pós-teste, principalmente G1. A esse respeito, convém ressaltar que já se esperava que G1 apresentasse melhores resultados no pós-teste, uma vez 
que o grupo foi submetido a um programa de promoção da criatividade, além de ter tido um tempo maior de interação com os pesquisadores, formando vínculos afetivos que podem afetar o desempenho, ou seja, variáveis afetivo-motivacionais também poderiam atuar nesse contexto (Tzuriel, 2001). Esses dados corroboram os efeitos dos programas de criatividade enquanto uma das formas de avaliação e desenvolvimento de habilidades criativas, conforme constatam pesquisas brasileiras sobre esses efeitos (Alencar, 1975; Pereira, 1996; Wechsler, 1987).

Em relação à avaliação cognitiva assistida (PBFD), os dados mostraram que, de modo geral, no pré-teste e no pós-teste, melhorou o desempenho das crianças na fase de assistência (aumento de pergunta de busca relevante e tentativa de solução correta), em relação à fase sem ajuda. Essa melhora de desempenho na elaboração de perguntas de busca, com restrição de alternativas, corroboram os resultados de investigações que constatam o efeito da avaliação mediacional (Avraham \& Ângelo, 1996; Burns \& cols., 1987, 1996; Carlson \& Wiedl, 1978). Essas evidências ainda confirmam uma das conclusões apontadas por Lidz (1992), na área de avaliação assistida, de que intervenções mediadas estão associadas a uma melhora em grande escala no desempenho de alunos mais comprometidos funcionalmente.

As diferenças favoráveis ao G2 e o simultâneo aumento interno do G1 respondem afirmativamente ao questionamento inicial sobre o uso de um instrumento com enfoque assistido para a avaliação de resultados de aplicação de um programa de promoção da criatividade em alunos com DA. O instrumento também se mostrou sensível para detectar diferenças entre os grupos até então não reveladas pelos TDE e WISC (instrumentos tradicionais), somente pelo Raven (instrumento tradicional).

Em outras palavras, o instrumento assistido, além de mostrar o desempenho dos grupos, permitiu visualizar as diferenças entre esses e, conseqüentemente, as diferenças individuais dentro dos grupos (perfil de desempenho). Também Ferriolli e cols. (2001) e Linhares, Santa Maria, Escolano e Gera (1998) observaram que diferenças individuais foram mais bem identificadas pela avaliação assistida.

Com base no exposto, nota-se a importância que a avaliação tem nesse contexto, vindo a beneficiar não somente o aluno, mas também os professores e o próprio planejamento curricular. Desse modo, as propostas de avaliação, assim como as de intervenção na área da criatividade estudadas nesta pesquisa, apresentam-se como tentativas práticas de abordagem de questões pertinentes ao nosso sistema educacional.

A proposta de uma avaliação assistida que leve em consideração o potencial do indivíduo, tem mostrado, através de pesquisa, o quanto crianças com diferentes problemas têm se beneficiado desse tipo de abordagem (Haywood \& Tzuriel, 2002; Linhares \& cols., 1998; Tzuriel, 2001; Sternberg \& Grigorenko, 2002).

O uso combinado de duas abordagens de avaliação (tradicional e assistido), com diferentes pressupostos teóricos subjacentes, pode a princípio parecer contraditório; mas, somente assim foi possível verificar a importância de ambas como instrumentos que permitem compreender melhor o aluno com DA, na medida em cada tipo de avaliação con- sidera diferentes aspectos do desempenho - o produto da aprendizagem, no caso da avaliação tradicional, não-assistida, e o processo de solução de problemas, no caso da avaliação assistida. $\mathrm{O}$ aluno pôde ser visto em diferentes perspectivas, isto é, foi possível avaliar o que aprendeu até o momento, o desempenho real comparado ao de seus pares (avaliação tradicional), bem como identificar seu modo de funcionamento cognitivo sob ajuda (avaliação assistida).

Com base no exposto acima, espera-se contribuir com outros estudos, que avaliem, com outras populações, a adequação de diferentes procedimentos de avaliação e seus instrumentos, mostrando que essas avaliações usadas em conjunto fornecem relevantes informações para o entendimento da questão, como defendem Linhares (1995) e Tzuriel (2001).

\section{Referências}

Alencar, E. M. L. S. (1975). Efeitos de um programa de criatividade em alunos de $4^{\mathrm{a}}$ e $5^{\mathrm{a}}$ séries. Arquivos Brasileiros de Psicologia, 27(4), 3-15.

Alencar, E. M. L. S. (1995). Criatividade. Brasília: Ed. Universidade de Brasília.

Alencar, E. M. L. S. (1996). A medida da criatividade. Em L. Pasquali (Org.), Teoria e Métodos de Medidas em Ciências do Comportamento (pp. 305-318). Brasília: MEC/INEP.

Alencar, E. M. L. S. (2000). O processo da criatividade: Produção de idéias e técnicas criativas. São Paulo: Makron Books.

Almeida, L. S. (1996). Considerações em torno da medida da inteligência. Em L. Pasquali (Org.), Teoria e Métodos de Medida em Ciências do Comportamento (pp. 199-223). Brasília: Laboratório de Pesquisa em Avaliação e Medida/Instituto de Psicologia/UnB:INEP.

Angelini, A. L.; Alves, I. C. B.; Custódio, E. M.; Duarte, W. F. \& Duarte, J. L. M. (1999). Manual Matrizes Progressivas Coloridas de Raven - Escala Especial. São Paulo: Centro Editor de Testes e Pesquisas em Psicologia.

Avraham, K. N. \& Angelo, D. (1996). The effects of feedback interventions on performance: A historical review, a metaanalysis, and a preliminary feedback intervention theory. Psychological Bulletin, 119(2), 254-284.

Bonamigo, E. M. R. (1980). Criatividade e ensino. Em J. C. Marques (Org.), Psicologia Educacional: Contribuições e desafios (pp. 222-253). Porto Alegre: Globo.

Burns, M. S.; Vye, N. J.; Bransford, J. D.; Delclos, V. \& Ogan, T. (1987). Static and dynamic measures of learning in young hadicapped children. Diagnostique, 12(2), 59-73.

Burns, M. S.; Delclos, V. R.; Vye, N. J. \& Sloan K. (1996). Changes in cognitive strategies in dynamic assessment. Em M. Luther; E. Cole \& P. Gamlin (Orgs.), Dynamic assessment for instruction: From theory to application (pp. 182-188). New York: Captus University Publications.

Carlson, J. S. \& Wiedl, K. H. (1978). Use of testing-the-limits procedures in the assessment of intellectual capabilities in children with learning difficulties. American Journal of Mental Deficiency, 82(6), 559-564.

Eysenk, H. J. (1999). As formas de medir a criatividade. Em M.A. Boden (Org.), Dimensões da criatividade. (P. Theobaldo, Trad., pp. 203-244), Porto Alegre: Artes Médicas.

Ferriolli, S. H. T.; Linhares, M. B. M.; Loureiro, S. R. \& Marturano, E. M. (2001). Indicadores de potencial de aprendizagem obtidos 
através da avaliação assistida. Psicologia: Reflexão e Crítica, 14(1), 35-43.

Fonseca, V. (1995). Introdução às dificuldades de aprendizagem. Porto Alegre: Artes Médicas.

García, J. N. (1998). Manual de dificuldades de aprendizagem: Linguagem, leitura, escrita e matemática. (J.H. Rodrigues, Trad.) Porto Alegre: Artes Médicas.

Gera, A. \& Linhares, M. B. M. (1998). Estratégias de perguntas de busca de informações na resolução de problemas de crianças com e sem queixa de dificuldade de aprendizagem [Resumo]. Em Sociedade Brasileira de Psicologia (Org.), Programas e Resumos da XXVIII Reunião Anual de Psicologia (p. 126). Ribeirão Preto: SBP.

Graham, S. \& Harris, K. R. (1989). The relevance of IQ in the determination of learning disabilities: Abandoning scores as decision makers. Journal of Learning Disabilities, 22(8), 500-512.

Guilford, J. P. (1968). Intelligende, creativity and their educational implications. San Diego, California: Kanapp, R. Publisher.

Haywood, H. C. \& Tzuriel, D. (2002). Applications and challenges in dynamic assessment. Peabody Journal of Education, 77(2), 40-63.

Jaben, T. H. (1986). Impact of instruction on behavior disordered and learning disabled students'creative behavior. Psychology in the Schools, 23, 401-405.

Lidz, C. S. (1992). Dynamic assessment: Some thoughts on the model, the medium, and the message. Learning and Individual Differences, 4(2), 125-136.

Linhares, M. B. M. (1995). Avaliação assistida: Fundamentos, definição, características e implicações para a avaliação psicológica. Psicologia: Teoria e Pesquisa, 11(1), 23-31.

Linhares, M. B. M.; Santa Maria, M. R.; Escolano, A. A. C. M. \& Gera, A. A. (1998). Avaliação assistida: Uma abordagem promissora na avaliação cognitiva de crianças. Temas em Psicologia, 6(3), 231-254.

Lubart, T. I. (1994). Criativity. Em R. Sternberg (Org.). Thinking and problem solving (pp. 289-332). New York: Academic Press.

Medeiros, P. C.; Loureiro, S. R.; Linhares, M. B. M. \& Marturano, E. M. A. (2000). Auto-eficácia e os aspectos comportamentais de crianças com dificuldade de aprendizagem. Psicologia: Reflexão e Crítica, 13(3), 327-336.

Neves, M. B. J. \& Almeida, S. F. C. (1996). O fracasso escolar na $5^{\mathrm{a}}$ série, na perspectiva de alunos repetentes, seus pais e professores. Psicologia: Teoria e Pesquisa, 12(2), 147-156.

Pereira, M. S. N. (1996). Efeitos de um treinamento de criatividade no desempenho escolar e nas habilidades criativas de crianças com dificuldades de aprendizagem. Dissertação de Mestrado, Universidade de Brasília, Brasília.

Pollack, S. N.; Pollack, D. \& Tuffli, C. S. (1973). Creativity in the severely retarded. The Journal of Creative Behavior, 1(1), 67-72.

Santa Maria, M. R. \& Linhares, M. B. M. (1999). Avaliação cognitiva assistida de crianças com indicações de dificuldade de aprendizagem escolar e deficiência mental leve. Psicologia: Reflexão e Crítica, 12(2), 395-417.

Siegel, L. S. (1989). Why we do not need intelligence test scores in the definition and analyses of learning disabilities. Journal of Learning Disabilities, 22(8), 514-518.

Stein, L. M. (1994). TDE - Teste de Desempenho Escolar: Manual para aplicação e interpretação. São Paulo: Casa do Psicólogo.
Sternberg, R. J. \& Grigorenko, E. L. (2002). Dynamic testing: the nature and measurement of learning potential. New York: Cambridge University Press.

Torgensen, J. K. (1988). Applied research and metatheory in the context of contemporary cognitive theory. Journal of Learning Disabilities, 21(5), 271-274.

Torgensen, J. K. (1989a). Cognitive and behavioral characteristics of children with learning disabilities: Concluding comments. Journal of Learning Disabilities, 22(3), 116-175.

Torgensen, J. K. (1989b). Why IQ is relevant to the definition of learning disabilities. Journal of Learning Disabilities, 22 (8), 484-486.

Torrance, E. P. (1965). Rewarding creative behavior: Experiments in classroom criativity. New Jersey: Prentice-Hall, Inc. Englewood Cliffs.

Torrance, E. P. (1976). Criatividade: Medidas, testes e avaliações (A. Arruda, Trad.). São Paulo: Ibrasa.

Torrance, E. P. (1990). Tests of creative thinking: Streamlined scoring guide figural and verbal $A$ and $B$. Bensenville, IL: Scholastic Testing Service, Inc.

Tzuriel, D. (2001). Dynamic assessment of young children. New York: Kluwer Academic/Plenum Publishers.

Virgolim, A. M.; Fleith, D. S. \& Pereira, M. S. N. (1999). Toc, Toc... Plim, Plim!: Lidando com as emoções, brincando com o pensamento através da criatividade. Campinas: Papirus.

Vygotsky, L. S. (1991). A formação social da mente: $O$ desenvolvimento dos processos psicológicos superiores $\left(4^{\mathrm{a}}\right.$ ed., J. Cipolla Neto, L. S. M. Barreto \& S. C. Afeche, Trads.). São Paulo: Martins Fontes. (Trabalho original publicado em 1934).

Wechsler, D. (1964). Escala de inteligência para crianças WISC - manual de aplicação e cotação (A. M. Poppovic, Trad.) Rio de Janeiro: CEPA. (Trabalho original publicado em 1949)

Wechsler, S. (1987). Efeitos do treinamento em criatividade em crianças bem-dotadas e regulares. Arquivos Brasileiros de Psicologia, 4, 95-110.

Wechsler, S. M. (1998). Criatividade: Descobrindo e encorajando. Campinas: Ed. Psy.

Wechsler, S. M. (2002). Avaliação da criatividade por figuras $e$ palavras - Testes de Torrance Versão Brasileira. Campinas: LAMP/PUCCamp. 